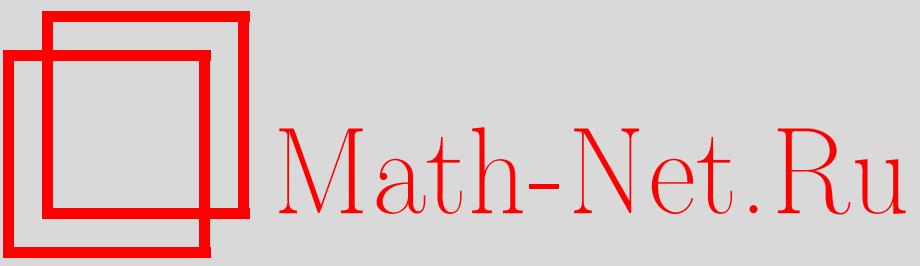

А. М. Вершик, Универсальное пространство Урысона, метрические тройки Громова и случайные метрики на натуральном ряде, УМН, 1998, том 53, выпуск $5,57-64$

DOI: https://doi.org/10.4213/rm69

Использование Общероссийского математического портала Math-Net.Ru подразумевает, что вы прочитали и согласны с пользовательским соглашением

http: //www . mathnet.ru/rus/agreement

Параметры загрузки:

IP: 34.229 .45 .116

26 апреля 2023 г., 14:59:12 


\section{УНИВЕРСАЛЬНОЕ ПРОСТРАНСТВО УРЫСОНА, МЕТРИЧЕСКИЕ ТРОЙКИ ГРОМОВА И СЛУЧАЙНЫЕ МЕТРИКИ НА НАТУРАЛЬНОМ РЯДЕ}

А. М. ВЕРШИК

СОДЕРЖАНИЕ

Введение

1. Универсальное пространство Урысона .......................... 58

2. Метрические тройки Громова ................................... 59

3. Случайные метрики на натуральном ряде и пространство Урысона . . 62

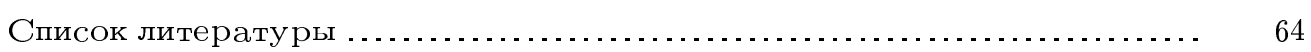

\section{Введение}

В одной из своих последних работ [1], увидевшей свет уже после неожиданной смерти автора, П.С. Урысон определил и изучил так назьваемое универсальное метрическое пространство. Эта работа не получила широкой известности, хотя того заслуживает. Я узнал о ней только в 1992 году во время работы над статьей [2], в которой была отмечена связь универсального пространства Урысона и расстояния Громова-Хаусдорфа между метрическими пространствами.

Как отметил П. С. Урысон, его работа была инициирована вопросом, заданньм ему М. Фреше, об универсальности какого-либо банахова или гильбертова пространства. Но построение П. С. Урысона оказалось более глубоким. На первьй взгляд, работа [1] выдержана в духе топологических увлечений тех лет, однако живая идея, содержащаяся в ней, выглядит весьма современно. Я еще раз вспомнил о ней во время обсуждений другой замечательной идеи М.Л. Громова, а именно, того, что ниже названо метрическими тройками, и обратил его внимание на пространство Урысона. В английском издании его книги [3] приведены некоторые новые соображения на этот счет. Цель настоящей заметки - проиллюстрировать связи этих двух тем между собой и соединить еще с одной вероятностной идеей, а также поставить некоторые задачи, связанные с этим кругом вопросов.

Работа частично поддержана грантом INTAS-94-3420. 


\section{1. Универсальное пространство Урысона}

Теорема [1]. Существует полное сепарабельное метрическое (=польское) пространство $U$, обладающее тремя свойствами.

1) (Универсальность) Всякое польское пространство мохет бить изометрично отображено в $U$.

2) (Однородность) Любые два конечных изометричных (=конгруэнтных) подмнохества пространства $U$ могут быть переведены одно в другое изометрией всего пространства $U$.

3) (Единственность) Свойства 1) и 2) определяют пространство U с точностью до изометрии однозначно.

Это пространство будем называть универсальным пространством Урысона. Построение пространства - несложно. П. С. Урысон строит его с помошњю прямой конструкции, начиная со счетного метрического пространства, универсального в классе конечных метрических пространств с рациональной метрикой, а затем пополняет его до $U$. Заметим, что универсальных пространств много; наиболее популярна замеченная после выхода работы П. С. Урысона С.Банахом и С.Мазуром универсальность пространства $C([0,1])$, но оно не является однородньм. Преимущество пространства Урысона, по сравнению с универсальньми банаховыми пространствами, видимо, недостаточно оцененное в литературе, (см., например, [4]) - в его единственности с точностью до изометрии и в максимально возможной транзитивности действия группы его изометрий. П. С. Урысон доказывает, что пространство $U$ связно и локально связно, как и сфера $S_{\varepsilon}(x)=\{y: \operatorname{dist}(x, y)=\varepsilon\}$ для любой его точки $x$; эта сфера является в том же смысле универсальньм пространством для всех польских пространств с диаметром, не превьшаюшим $2 \varepsilon$. Как отмечает редактор двухтомника сочинений П. С. Урысона (П. С. Александров) в примечаниях к работе [1], было бы хорошо дать более явную реализацию этого важного геометрического объекта. Мы делаем далее некоторый шаг в этом направлении, а пока сделаем несколько замечаний, связанных с понятием универсального пространства Урысона. Сначала очевидные следствия, соединяющие его с более поздними понятиями.

СлЕДСтВИЕ. 1) Пространство $U$ является пространством Александрова (А.Д.), в том смысле, что расстояние межсду любыми двумя точками есть инфимум длин спрямляемьх кривьх, их соединяющих (фактически әто отмечено ужсе в работе П. С. Урысона).

2) Расстояние Громова-Хаусдорфа (см. [3]) между метрическими пространствами $X$ и $Y$ можсет вычисляться по формуле

$$
d(X, Y)=\inf \{\tilde{\rho}(i(X), j(Y))\}
$$

где inf берется по всем вложсениям $i, j$ пространств $X, Y$ соответственно в пространство Урысона, а $\tilde{\rho}$-метрика на подмнохсествах (метрика Хаусдорфа) этого пространства (см. [2]).

Из $n$-кратной при любом натуральном $n$ изометрической транзитивности действия группы $G(U)$ - изометрий пространства $U$ - вытекает, что это очень богатая и интересная группа. 
Вопрос. Описать группу $G(U)$. Какие у нее имеются линейнье представления? Какова ее алгебрачческая и топологическая структура? Есть ли у нее естественный инфинитезимальный аналог?

Эти вопросы, по-видимому, ранее не обсуждались.

Вьше говорилось о сфере в пространстве Урысона как об универсальном однородном пространстве в классе польских пространств с диаметром не вьше фиксированного. Точно так же можно поставить вопрос об универсальном однородном пространстве, например, для метрических компактов, $\varepsilon$-энтропия которых не выше заданного роста, и т. д.

\section{2. Метрические тройки Громова}

Классификация польских пространств с точностью до изометрии - необозримая задача. Более точно, эта классификация не является в современной терминологии “гладкой" - см. [10]. Предположим, что на польском пространстве $(X, \rho)$ задана произвольная борелевская вероятностная мера $\mu$. По известной теореме (В.А. Рохлин, $[5])$ пара $(X, \mu)$ изоморфна в смысле теории меры стандартному пространству с мерой (пространству Лебега). В частности, если эта мера непрерьвна, то всякое польское пространство с борелевской вероятностной мерой изоморфно отрезку [0,1] с лебеговой мерой. Оказывается, между необозримой классификацией польских пространств $(X, \rho)$ с точностью до изометрии и тривиальной классификацией пар $(X, \mu)$, как пространств с мерой, лежит удачная промежуточная задача, предложенная М. Л. Громовым, о классификации метрических пространств $(X, \rho, \mu)$ с борелевской вероятностной мерой с точностью до изометрий, сохраняющих меру. Эта классификация оказалась обозримой, в отличие от первой, и нетривиальной, в отличие от второй классификации. Она и составляет теорему Громова, к которой мы и переходим, слегка изменив ее форму. Назовем метрической тройкой польское пространство $X$ с метрикой $\rho$ и борелевской вероятностной мерой $\mu-(X, \rho, \mu)$. В дальнейшем, не умаляя обшности, будем всегда считать, что носитель меры есть все пространство (т.е. все открытые непустые множества имеют положительную меру). Две тройки $(X, \rho, \mu)$ и $\left(X^{\prime}, \rho^{\prime}, \mu^{\prime}\right)$ метрически (в смысле и метрики, и меры, - в русской литературе слово “метрически" употребляется в обоих смыслах, но здесь смысл общий) изоморфны, или просто изоморфны, если существует изометрия, отображающая первое пространство на второе и переводяшая меру $\mu$ в меру $\mu^{\prime}$. Этот тип изоморфизма, как проиллюстрировано в [3], полезен в спектральной геометрии, а также в эргодической теории и, особенно, в асимптотических задачах. Но главное в том, что соответствующая классификация оказьвается весьма конструктивной. Построим полную систему инвариантов метрических троек относительно этого изоморфизма. Рассмотрим множество $R$ бесконечных матрии, расстояний, т.е. симметричных матриц с вешественными неотрицательньми коэффициентами $r_{i, j}, i, j \in \mathbb{N}$, равными нулю на главной диагонали и удовлетворяюшими неравенству треугольника:

$$
r_{i, j}+r_{j, k} \geqslant r_{i, k}
$$

(не требуется, чтобы внедиагональные элементы были отличны от нуля). Множество $R$ есть вьпукльй конус в векторном пространстве всех бесконечных, вещественных, 
симметричных матриц, замкнутый в слабой топологии этого пространства. Пусть $X^{\infty}$ - произведение счетного числа копий пространства $X$. Отображение $\phi: X^{\infty} \rightarrow$ $R$ сопоставляет каждой счетной последовательности $\left\{x_{i}\right\} \in X^{\infty}, i \in \mathbb{N}$, матрицу расстояний $\left(\rho\left(x_{i}, x_{j}\right)\right), i, j \in \mathbb{N}$. Введем на $X^{\infty}$ продакт-меру $\mu^{\infty}$ с сомножителем $\mu$, и пусть $M_{\mu}$ есть $\phi$-образ этой меры на пространстве $R$.

Teоpema (см. [3]). Две метрические тройки $(X, \rho, \mu)$ u $\left(X^{\prime}, \rho^{\prime}, \mu^{\prime}\right)$ изоморфнь тогда и только тогда, когда мерь $M_{\mu}$ и $M_{\mu}^{\prime}$ на $R$ - совпадают.

Таким образом, классификация метрических троек по Громову - “гладкая", т.е. классы изоморфных троек имеют хорошую параметризацию, а пространство классов есть множество некоторых мер на $R$.

ДокАЗАТЕльство. Если тройки изоморфин, то совпадение мер $M_{\mu}$ и $M_{\mu}^{\prime}$ - очевидно. Нетривиальна достаточность совпадения, которая мгновенно следует из эргодической теоремы. Действительно, если $M_{\mu}$ и $M_{\mu}^{\prime}$ совпадают, то существует хотя бы одна матрица из $R$ (на самом деле таковы почти все матрицы по этой мере), прообраз которой в $X^{\infty}$ (соответственно в $\left.X^{\prime \infty}\right)$, в силу эргодической теоремы (закона больших чисел), есть равномерно распределенная в $X$ относительно $\mu$ (соответственно в $X^{\prime}$ относительно $\mu^{\prime}$ ) всюду плотная (поскольку меры имеют полный носитель) последовательность. Эти две последовательности, как метрические пространства, изометричны, так как их матрищы расстояний совпадают; изометрия продолжается до изометрии пополнений, а их равномерная распределенность гарантирует нужное сохранение мер при изометрии, что и требовалось доказать.

Опишем теперь условия на меры $M_{\mu}$ на множестве $R$, возникаюшие как образы при отображениях $\phi$. Прежде всего, они инвариантны относительно действия на $R$ бесконечной симметрической группы $S_{\infty}$, одновременно переставляющей строки и столбцы матриц, поскольку они представляют собой $\phi$-образы продакт-мер, инвариантных относительно всех (не только финитных) перестановок. По той же причине меры $M_{\mu}$ әргодичны относительно этого действия. Но этих условий недостаточно. Например, точечная мера, сосредоточенная на матрице, у которой вне главной диагонали стоят единицы, а на ней - нули, отвечает обоим условиям, но, с одной стороны, - это матрища расстояний счетного дискретного пространства, расстояние между любыми двумя различными точками которого равно единице, а в то же время, дельта-мера может отвечать только одноточечному пространству.

Другое необходимое условие, которому удовлетворяют все меры $M_{\mu}$, состоит в следуюшем:

для всякого $\varepsilon>0$ сушествует такое натуральное $N$, что

(*) $\quad M_{\mu}\left\{r \in R: \lim _{n \rightarrow+\infty} \frac{\#\left\{j: 1 \leqslant j \leqslant n, \min _{1 \leqslant i \leqslant N} r_{i, j}<\varepsilon\right\}}{n}>1-\varepsilon\right\}>1-\varepsilon$.

Это свойство меры $M_{\mu}$ вытекает из известной теоремы о том, что для всякой вероятностной меры в польском пространстве существует сигма-компакт полной меры (см. [6]). Если неравенство для предела, стоящее в скобках в условии (*), заменить на неравенство для всех достаточно больших $j$, т.е. наложить условие: 
для всякого $\varepsilon>0$ сушествует такое натуральное $N$, что

$$
M_{\mu}\left\{r \in R: \text { для любых } j>N \min _{1 \leqslant i \leqslant N} r_{i, j}<\varepsilon\right\}>1-\varepsilon
$$

то мы получим необходимое и достаточное условие компактности пространства $X$. Достаточность доказывается просто: матрищы расстояний, удовлетворяюшие условию в скобках, отвечают последовательностям, у которых первые $N$ элементов образуют $\varepsilon$-сеть. Поскольку мера таких матриц положительна при всех $\varepsilon$, то пространство содержит конечную $\varepsilon$-сеть при любом $\varepsilon$. Необходимость - очевидна.

Точно так же, но уже с помощью эргодической теоремы, доказьвается общая

ТЕОРемА. Всякая борелевская вероятностная мера $M$ на множестве матрич расстояний $R$, инвариантная и эргодическая относительно действия $S_{\infty}$ и удовлетворяющая условию (*), совпадает с мерой $M_{\mu}$ для некоторой метрической тройки $(X, \rho, \mu)$.

Множество всех мер, описанных теоремой, обозначим через $\mathbb{M}$. Оно, таким образом, и служит множеством классов изоморфизма метрических троек.

Введем слабую топологию на пространстве мер $\mathbb{M}$, индуцированную слабой топологией на $R$. Из предыдущего следует, что тем самым введена топология в пространстве классов изоморфизма метрических троек. Возможно и более полное описание мер из множества М. Заметим, например, что матричные элементы (стояшие выше главной диагонали), вообще говоря, не являются независимыми относительно мер $M \in \mathbb{M}$ - в этом легко убедиться, рассмотрев совместное распределение трех элементов $r_{1,2}$, $r_{1,3}, r_{2,3}$, подчиняющихся неравенству треугольника. Более того, элементы одной строки (например, первой) также не являются, вообше говоря, независимыми, так как соответствующая одной строке мера $-S_{\infty}$-инвариантна, но не эргодична. Поучительно рассмотреть, например, меру $M_{\mu}$, соответствуюшую единичному кубу в $\mathbb{R}^{n}$, снабженному лебеговой мерой и евклидовой метрикой. Еще интересней дать описание меры $M_{\mu}$ для тройки $(C[0,1], \rho, w)$, где $\rho$ - обьгная метрика, порожденная $\max$-нормой на $C[0,1]$, а $w$ - винеровская мера. Все это дает новые примеры эргодических, инвариантных относительно симметрической группы мер на пространстве бесконечных матриц. Имеются более глубокие свойства независимости и асимптотической независимости мер из М. Упомянем попутно, что описанию мер, инвариантных относительно того или иного действия симметрической группы, мер на пространстве бесконечных матриц, вне связи с рассматриваемым здесь вопросом, посвяшен ряд работ см. [8], [9]; описание в [9] - неполно, хотя метод отыскания мер (эргодический метод), изложенньй там, применим и к нашему случаю. Подобные задачи имеют применения в теории вероятности и комбинаторике. Инвариантные и эргодические относительно действия симметрической группы меры на матрицах расстояний можно было бы назвать “виртуальньми метрическими тройками”. Очень интересен вопрос о поведении спектров случайных матриц расстояний, распределенных в соответствии с такими мерами или мерами из $\mathbb{M}$.

Функция $N(\varepsilon)$, минимизирующая при заданном $\varepsilon$ число $N$ в условии $(*)$, есть не что иное, как $\varepsilon$-энтропия метрического пространства с мерой (т.е. минимальное число точек, объединение $\varepsilon$-окрестностей которых имеет меру не меншшю, чем $1-\varepsilon$ ), 
рассмотренная еше в первых работах А.Н. Колмогорова по теории информации [7] и многократно переоткрьвавшаяся позже.

На меру $M_{\mu}=\phi\left(\mu^{\infty}\right)$ можно также смотреть как на инвариант метрики $\rho$, как функции от двух переменных на пространстве $X \times X$ относительно одновременного действия (на каждый из аргументов) групшы преобразований пространства с мерой $(X, \mu)$, сохраняюших меру $\mu$.

Задача о метрической (в смысле теории меры) классификации измеримых функций одного аргумента решена В. А. Рохлиным, полной системой инвариантов служат распределение функции и инварианты разбиения на прообразы точек; однако классификация функций двух и более переменных остается не решенной в целом. Случай метрик - особый, здесь, как мы видели, полным инвариантом классов служит совместное распределение парных расстояний независимых счетных систем точек. Совместные распределения конечных наборов матричных элементов относительно мер доставляют конечно-частичные инварианты, которых, вообше говоря, недостаточно для восстановления всей меры.

Обший план исследований (см. [3]) состоит в том, чтобы по мере $M_{\mu}$ заключать о тех или иных инвариантных относительно изоморфизма свойствах тройки $(X, \rho, \mu)$ или о свойствах $(X, \rho)$ и, наоборот, задавая меру $M_{\mu} \in \mathbb{M}$, строить пространства с заданными свойствами. Кроме того, вводя ту или иную топологию или метрику на мерах из $\mathbb{M}$, можно изучать аппроксимационные и асимптотические свойства метрических троек, точнее, классов их метрического изоморфизма. В качестве одного из примеров мы применяем ниже этот подход к пространству Урысона.

\section{3. Случайные метрики на натуральном ряде и пространство Урысона}

Взглянем на наше построение с иной стороны. Всякая матрица расстояний порождает (полу)метрику на натуральном ряде, а пополнение по ней дает польское пространство. Если матрица - случайна, то и метрика на натуральном ряде - случайна. Но если распределение этих матрищ, т.е. мера $\mu$ на $R$, принадлежит $\mathbb{M}$, мы получаем случайные метрики на натуральном ряде, соответствующие случайному выбору различных счетных всюду плотных множеств одного и того же пространства, причем элементы этих множеств выбираются независимо и с одним и тем же распределением. Посмотрим, как получить таким образом пространство Урысона, снабженное мерой. Описать матрицу расстояний для какой-нибудь счетной всюду плотной последовательности в пространстве Урысона - и значит определить это пространство, что сделано в [1]. Но можно задавать не просто одну матрицу расстояний, т.е. не одно, ничем не выделенное счетное всюду плотное множество будущего пространства, а описьвать случайное плотное множество относительно некоторой меры с полным носителем в этом пространстве, т.е. меру $M \in \mathbb{M}$ на $R$ или класс метрического изоморфизма, соответствуюший тройке $(U, \rho, \mu)$, где $(U, \rho)$ - пространство Урысона, $\mu-$ мера с полным носителем на нем. Произвольность выбора плотного счетного множества в $(U, \rho)$ заменяется произвольностью выбора меры на нем с полньм носителем, задающей счетное множество статистически. Но при этом мы видим новые интересные свойства пространства.

Теорема. Пусть $(X, \rho, \mu)$ - метрическая тройка и $M \in \mathbb{M}$ - соответствующая ей мера на $R$ - пространстве всех бесконечных матрии расстояний. Если 
для произвольных $n$, матрицы расстояний $D=\left(d_{k, s}\right), k, s=1, \ldots, n$, порядка $n$ и положительного є существует такой номер $N$, что

$M\left\{r \in R\right.$ : существуют такие натуральные $i_{1}, i_{2}, \ldots, i_{n}<N=N(D, \varepsilon)$,

$$
\text { что } \left.\left|r_{i_{s}, i_{k}}-d_{k, s}\right|<\varepsilon, k, s=1, \ldots, n\right\}>1-\varepsilon,
$$

то $(X, \rho)$ есть пространство Урысона, а $\mu$-мера с полнылм носителем на нем. Обратно, мера $M_{\mu}$, отвечающая тройке $(U, \rho, \mu)$, әде $(U, \rho)$ - пространство Урыссона, ан-мера на нем с плотным носителем, обладает этим свойством.

Доказательство этой теоремы сводится к доказательству следующего варианта основной леммы, доказанной в [1]: пополнение счетного метрического пространства $(Y, \rho)$ есть пространство Урысона тогда и только тогда, когда для любых его точек $x_{1}, x_{2}, \ldots, x_{n} \in Y$, любого числа $\varepsilon$ и любой матрицы расстояний $D=\left(d_{i, k}\right)$ порядка $n+1$, у которой $d_{i, k}=\rho\left(x_{i}, x_{k}\right), i, k=1,2, \ldots, n$, сушествует такая точка $x_{n+1} \in Y$, что элементы матрищы $D^{\prime}$ расстояний точек $\left(x_{1}, x_{2}, \ldots, x_{n+1}\right)$ отличаются (в последнем столбце) от элементов матрицы $D$ не более чем на $\varepsilon$.

Из этой теоремы и инвариантности мер $M_{\mu}$ относительно $S_{\infty}$ вытекает такое утверждение.

СлЕДСТВИЕ 1. Носитель меры $M_{\mu} \in \mathbb{M}$, отвечающей метрической тройке $(X, \rho, \mu)$, совпадает со всем $R$ тогда и только тогда, когда $(X, \rho)$ - универсальное пространство Урысона, а $\mu$-борелевская вероятностная мера на нем с полным носителем.

Совпадение носителя (т.е. минимального слабо замкнутого множества полной меры) меры $M_{\mu}$ со всем пространством $R$ означает, что для любого натурального $n$, всякой матрицы расстояний $D=\left(d_{i, k}\right)$ порядка $n$ и любого положительного $\varepsilon$

$$
M_{\mu}\left\{r \in R:\left|r_{i, k}-d_{i, k}\right|<\varepsilon, i, k=1, \ldots, n\right\}>0
$$

Из этого следствия и свойств множества мер с полным носителем на метрическом пространстве вытекает

СЛЕДСТВИЕ 2. В пространстве классов метрических троек классы троек $(U, \rho, \mu)$, где $U$ - пространство Урысона, образуют всюду плотное $G_{\delta}$-множсесmво.

Тем самым, среди классов изоморфизма метрических троек классы троек $(X, \rho, \mu)$ с пространством Урысона в качестве $X$ являются типичными. Группа изометрий $G(U)$ пространства Урысона $U$ действует на пространстве мер на нем, т.е. на меpax на $\mathbb{M}$ с полным носителем. Это действие представляет интерес с точки зрения изучения группы $G(U)$. Заметим еше, что инвариантное описание метрической тройки, в которой пространство обладает свойством универсальности, но без урысоновской однородности (как в примерах Фреше-Банаха-Мазура), видимо, не имеет столь простой формулировки, подобной следствию 1. Задание мер на метрическом пространстве с помощью совместных распределений попарных расстояний (т.е. с помощью мер $M_{\mu}$ ) не является традищионньм и поднимает ряд новых вопросов, в частности: 
Вопрос. Дать явное описание мер с полным носителем на пространстве Урысона с помощью теоремы, приведенной выще; иначе говоря, описать непосредственно меру $M_{\mu}$ на $R$ как инвариант тройки $(U, \rho, \mu)$, где $\mu$ - какая-либо мера с полным носителем на $U$. Существует ли какой-либо аналог винеровской или гауссовских мер на пространстве Урысона?

Последний вопрос сводится к определению аналога гауссовских мер на выпуклом конусе $R$ матриц расстояний; здесь может быть полезньм эргодический метод [9], которьй уже использовался в подобных задачах.

В заключение заметим, что в духе предыдуших рассмотрений уместно непосредственно использовать матрицы расстояний в эргодической теории и теории динамических систем, например, следуюшим образом. Пусть в метрическом пространстве $(X, \rho)$ задан минимальный строго эргодический гомеоморфизм $T$. Тогда по бесконечной матрице расстояний любой из его орбит $-\left(T^{n} x, n \in \mathbb{Z}\right)$ можно, как и вьше, восстановить всю систему, т.е. пространство $(X, \rho)$ и гомеоморфизм $T$, а также единственную инвариантную относительно этого гомеоморфизма меру $\mu$. Точно также определяется эргодическая мера на пространстве матрищ расстояний $R$, т.е. случайная матрица расстояний. Эта мера, как и ранее, является образом при отображении $X^{\infty} \rightarrow R$ некоторой стационарной (т.е. инвариантной относительно двустороннего сдвига) меры на $X^{\infty}$, но уже, вообше говоря, не являюшейся продакт-мерой, как было до сих пор. Таким способом по одной орбите и ее матрице расстояний можно получать любые инварианты динамических систем в метрическом пространстве.

\section{СПИСОК ЛИТЕРАТУРЫ}

[1] Urysohn P.S. Sur un espace metrique universel // Bull. Sci. Math. 1927. V. 51. P. 1-38; рус. перев.: Урысон П.С. Об универсальном метрическом пространстве // В сб. П. С. Урысон, Труды по топологии, т. 2. М.: ГИТТЛ, 1951.

[2] Berestovsky V.N., Vershik A. M. Manifolds with intrinsic metric, and nonholonomic spaces // Representation Theory and Dynamical Systems / ed. A. M. Vershik. Providence, RI: Amer. Math. Soc., 1992. P. 253-267.

[3] Gromov M. Metric Structures on Riemannian and Non-Riemannian Spaces. Basel-Boston: Birkhäuser, 1998.

[4] Люстерник Л. А., Соболев В. И. Элементы функционалшного анализа. М., 1951.

[5] Рохлин В.А. Об основных понятиях теории меры // Матем. сб. 1949. Т. 25 (67). № 1. C. $107-150$.

[6] Халмош П. Теория меры. М.: ИЛ, 1950.

[7] Колмогоров А.Н. Теория передачи информации // А.Н. Колмогоров. Теория информации и теория алгоритмов. М., 1987. С. 29-58.

[8] Aldous D. J. Exchangeability and related topics // Lecture Notes in Math. 1985. V. 1117. P. 1-198.

[9] Вершик А. М. Описание инвариантных мер для действий некоторых бесконечномерных групп // Докл. АН СССР. 1974. Т. 218. № 4. С. 749-752.

[10] Kechris A. Actions of Polish Groups and Classification Problems // Preprint Caltech., 1997. 